\title{
Automation of FMET \& Regression Tests for Doser After-Treatment Controls using Load-box User-Interface-System Hardware-In-Loop Bench
}

\author{
Priya Malani $^{1}$, Chidambar Rao Bhukya ${ }^{2}$, Jagdish Vishwasrao ${ }^{3}$, Jayesh Sonkusare ${ }^{4}$ \\ M.Tech. Electronics Engineering, Veermata Jijabai Technological Institute, Mumbai, India ${ }^{1}$ \\ Cummins Emission Solutions, Cummins Technologies India Pvt. Ltd, Pune, India ${ }^{2,3,4}$
}

\begin{abstract}
Hardware-in-loop (HIL) simulation is a technique which allows the development and testing of Electronic Control Module (ECM) virtually in the real time domain in order to analyse or overlook the behaviour \& performance of various sensors and actuators/loads present inside the Doser. Testing done by using the HIL approach reduces the overall cost, manual efforts and overall testing time as compared to manual testing to a greater extent. This paper describes the case study to automate the Failure Mode Effects Tests (FMET) and Regression Tests of Doser System where a HIL technique is invoked for the authentication and affirmation of the performance of the Doser. The system is developed by using Load-Box User Interface System (LUIS) hardware and Digital FMET box which auto-simulate fault conditions for various sensors like Pump Unit Temperature Sensor, Mixed Air Pressure Sensor, NOx sensor, Selective Catalyst Reduction Inlet (SCR) Temperature Sensor and actuators like Internal Metering Valve (IMV), Bypass Valve (BPV), Air Shutoff Valve (ASOV). The conventional manual FMET testing is time-consuming and prone to various manual errors. Thus to reduce the time and manual errors and to increase the overall efficiency the fully automated test bench is developed. The test bench uses National Instruments (NI) Test stand for writing test sequence scripts to fully automate the manual FMET and regression tests. The developed automated test bench deliver faster test results with reduced manual errors, reduced man power and improved cost saving.
\end{abstract}

Keywords: After-Treatment System, Hardware-In-Loop (HIL) Testing, Load Box User Interface System (LUIS), Digital FMET Box, FMET, Regression Tests.

\section{INTRODUCTION}

The On-Highway vehicles like Heavy Duty vehicles, Medium Duty vehicles \& Low Duty vehicles consists of HighHorse Power engines. Thus, exhaust gases coming out from these vehicles consist of Oxides of Nitrogen; NOx, Sulphur; SOx and Particulate Matter; PM. It is required to fulfil the Bharat Stage norms decided by the government to regulate air pollution levels. Therefore, After-treatment system is required to control the pollution due to the exhaust gases coming out of these high power engines. Thus, with the help of Doser, Diesel Exhaust Fluid (DEF) i.e. Urea solution is sprayed over the exhaust gases to convert NOx into atmospheric Nitrogen and Water. DEF solution consist of $32.5 \%$ of urea and $67.5 \%$ of water concentrations. The amount of Urea to be sprayed is calculated by ECM according to the feedback given from the Doser System. Thus to make the Doser System free from any fault conditions, various sensors and loads present inside it are to be tested.

In recent years, Hardware-In-Loop testing has been proved to serve as an extensive rapid model and automated testing platform. HIL testing can be applied to a wide variety of fields mainly, Aerospace, Automotive, Power System, Digital Electronics, Robotics etc.., HIL testing is a technique that replaces the physical model with the mathematical illustration that completely describes the important features of the physical model.

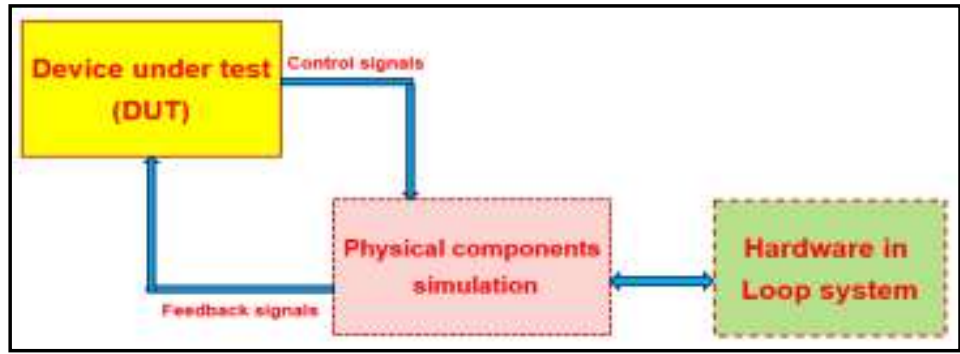

Figure-1 Generalized Block diagram of HIL system 


\section{ISO 3297:2007 Certified \\ Vol. 5, Issue 5, May 2017}

International Journal of Innovative Research in Electrical, Electronics, Instrumentation and Control Engineering

HIL testing environment provides a rapid prototyping platform for the development and testing of the various components such as sensors and actuators or loads which are present inside the Doser which are used for the after treatment purpose. Figure-1 shows the generalised block diagram of Hardware-In-Loop system.

In the figure-1, it is shown that a device under test such as ECM interfaces directly with the real time computational hardware such as LUIS box that compute the responses i.e. fault conditions of the sensors and loads of the physical component system i.e. Doser. Therefore, the fully automation of FMET tests and Regression tests from manual testing method increases the overall cost, overall time, reduces manual efforts and manual errors thus increasing the overall efficiency and effectiveness.

\section{FMET \& REGRESSION TEST OF CONTROLS SOFTWARE}

Each controls software logic which is newly developed, updated or modified as per customer requirements/feedback, its performance is required to be validated as per expectations by conducting FMET \& Regression Tests in the controls lab before it is released for field. As part of these tests, the software performance is analysed by purposefully inputting the noise conditions into the system manually viz. tampering, obstructing and damaging both physically and functionally. In these noise conditions also, the control logics have to perform as per expectations. The simulation of noise conditions is also called as Fault conditions Simulation, which have various types \& forms as described below:

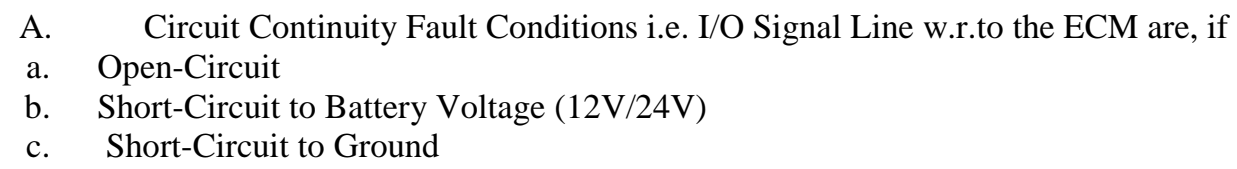

B. Functionality Fault Conditions:

a. As part of Functionality Fault Simulations, the loads Like ASOV, BPV and IMV have to be forcefully control through external supply of voltage bypassing ECM commands. In this, it is manually required to physically open the existing connections of corresponding valves and connect second external voltage supplies/connectors.

b. Similarly, performance of the controls software is tested against tampering of the corresponding Air-Intake, DEF Suction and DEF Transfer Lines viz. full \& partial blockage, Open and broken lines, done through manual simulation.

c. The DEF Nozzle have to be opened completely from the transfer line, with manual efforts.

\section{Regression Tests:}

a. Pressure Control Tests: which requires the DEF dosing rate to be changed manually for every 2 hours in the total 9 hours of the test requirements.

\section{III.CONVENTIONAL MANUAL TESTBENCH SETUP}

Earlier, the FMET and Regression Tests are conducted on Manual Test Benches where an engineer is required to manually simulate the above Fault Conditions,

a. Using a 'Manual Breakout Box' by making/breaking electrical circuits using jumper wires for each Pin of the ECM corresponding to each Fault Code,

b. Using the mechanical On / Off Valves for Air and DEF Flow Control.

c. Manually removing/connecting the corresponding ASOV, BPV, IMV and Nozzle connections.

Approximately there are 50 Fault Codes/conditions per an ECM. Likewise, for the UA2 Dosing System, there are 4 different types of ECMs available. There are 4 release cycles in a year, i.e. Total Number of different FMET \& Regressions tests required to be conducted per UA2 Dosing System in a year is approx. (4 ECMs $\times 4$ Quarters $=16$ FMETs). Total numbers of Fault Codes in a year to be simulated in UA2 Dosing System approx. are: (4 ECMs $\times 4$ Quarters $\times 50$ Fault Codes $)=800$ Fault Codes per year. Therefore, test duration in manual test bench is approximately 550 hours per annum. Also, the system is prone to various types of manual errors. Like, parallax (e.g. value reading, selection of voltage levels, urea dosing rates etc.), Test Engineer person to person variations and judgments e.g. valves rotation, connection timings, and tuning timings. Comparatively more chances of damaging sensors and actuators due to person to person judgement. Data logs recording in the Tuning Software sometimes may be overlooked by the test engineer and needs test repetition. Figure-2 shows the Block diagram of manual test bench of UA2 Doser. Therefore, in order to overcome above difficulties involved in Manual Testing and to improve overall effectiveness and efficiency of time \& cost, development of the Fully Automated FMET and Regression Test Bench is done. 
Vol. 5, Issue 5, May 2017

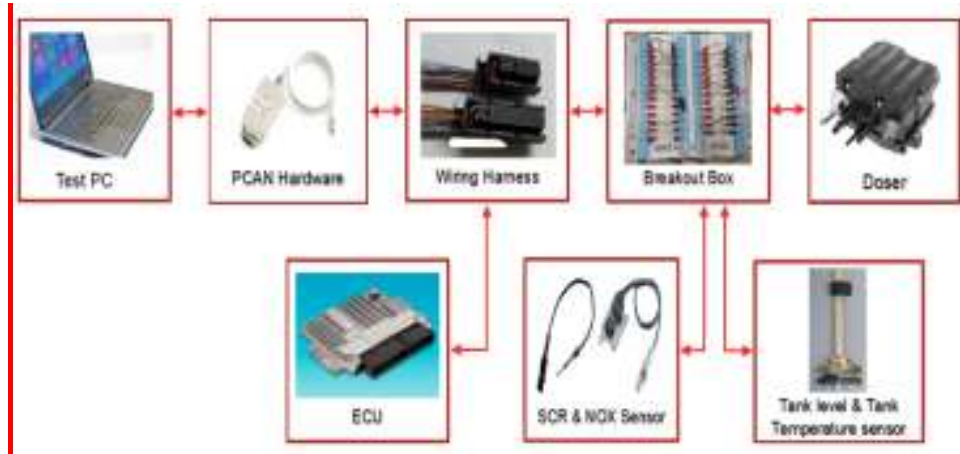

Figure-2 Block diagram of Manual test bench setup for UA2 Doser

\section{HIL SYSTEM DEVELOPMENT FOR AUTOMATED TESTING}

The approach to fully automate the FMET and Regression test using Hardware in loop test bench platform for the simulation of various sensors and actuators present inside the doser is done by the Load box user interface system and its interfacing with the Integrated Circuits Board. In addition to these writing of the test sequence scripts is done by using NI Teststand.Figure-3 shows the Block diagram of HIL system.

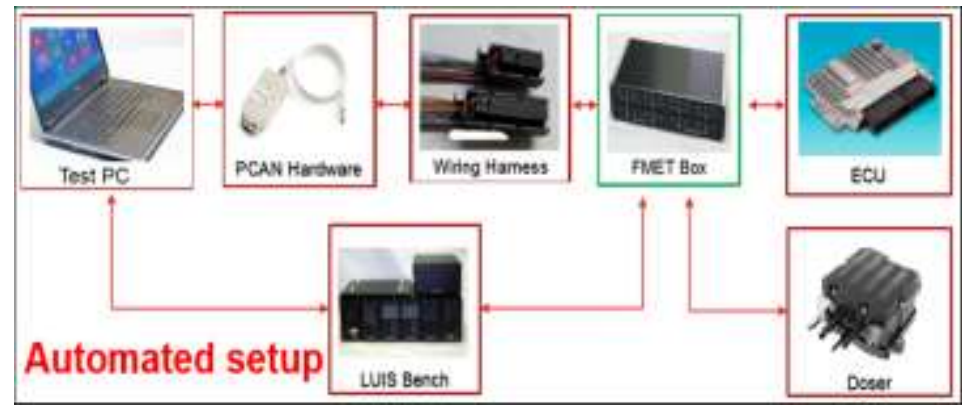

Figure-3 Block diagram of the HIL system

The developed system consists of LUIS box, ECU, Doser, Digital FMET box. The connection between the LUIS box, digital FMET box, ECM and, Doser is done by wiring harness. Digital FMET box is used as the replacement of manual breakout box. CAN adapter is used for the communication between Doser and ECM. \& between user computer and ECM and LUIS box. The blocks of the above block diagram is described below:

A. Load Box User Interface System: - Load box system is a bench top engine simulator system. It is used for the open loop testing purpose i.e. it facilitates for the bench top engine hardware and software testing. LUIS box consists of various modules like Main module, Analog module, Resistive load module, Wave maker module, Switch module. The simulation of various conditions of sensors and loads of Doser are simulated with the help of the respective modules of the LUIS box.

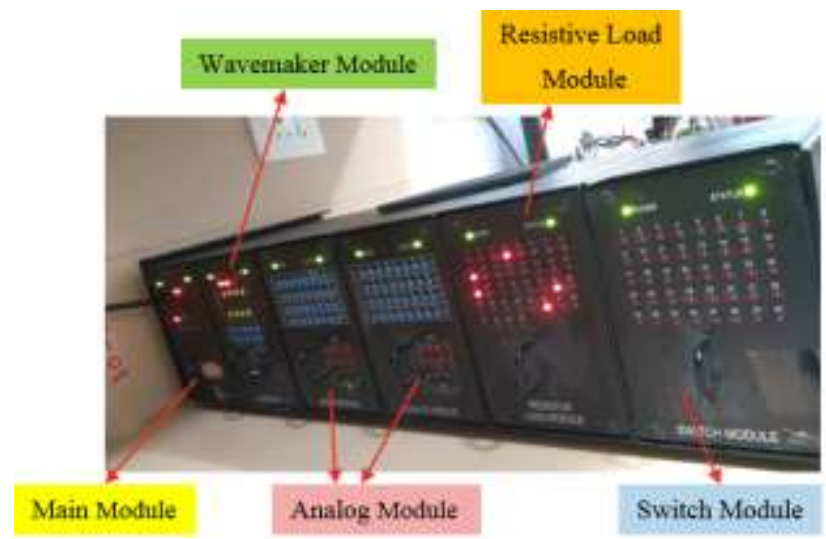

Figure-4 LUIS GEN2 Box 


\section{IJIREEICE \\ Vol. 5, Issue 5, May 2017}

B. Digital FMET Box: - The Digital FMET box has electronic controlled relays in it which can auto simulate circuit continuity fault conditions. The Electrical Circuit Continuity Fault Conditions viz. Open-Circuit, Short-Circuit to Battery Voltage and Short-Circuit to Body Ground can be achieved with the help of Digital FMET Break-out Box.The relay pin mapping of the relay boards of digital FMET box is defined in the Software Architecture tool table.

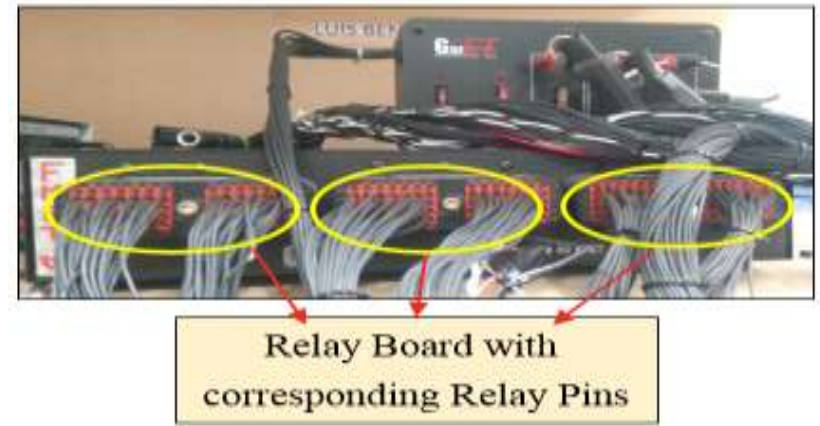

Figure-5 Digital FMET Breakout Box

C. Electric Control Module: - Electronic control module (ECM) is like central processing unit, which is responsible for all the decision making operations and the controlling operations required in the system. Simulation of any fault code/condition is detected by the ECM \& thus if the fault code is generated it transmit it over CAN which is connected with the test PC to show the corresponding Active \& Inactive status of the fault.

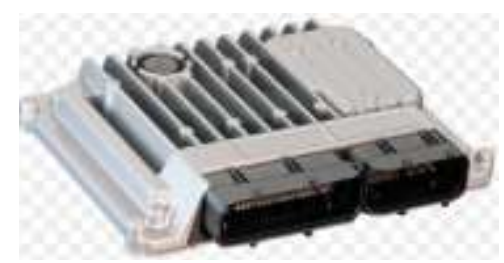

Figure-6 Electronic control module

D. Doser: - Doser is the heart of the After-treatment system. Doser consists of various types of sensors and Actuators within it, such as Pump unit temperature sensor, mixed air pressure sensor, DEF Pump-Pressure Sensor, Pump Motor and various actuators like Bypass Valve, Metering Valve, and Air Shutoff Valve. Doser is used for spraying DEF with certain rates over the NOx in the Exhaust Gas Processor to convert it into atmospheric $\mathrm{N}_{2}$ and $\mathrm{H}_{2} \mathrm{O}$.

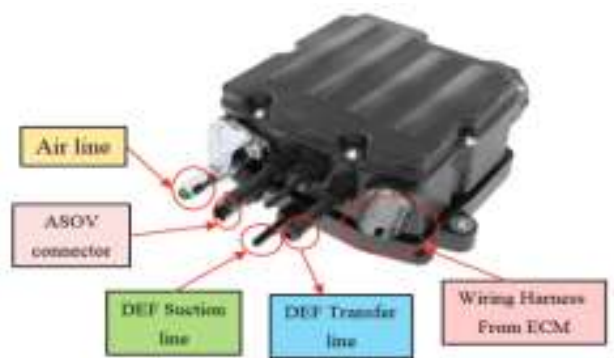

Figure-7 Urea Air assisted Doser

E. CAN Adapter: - USB/CAN is used to provide communication between the Doser, ECM, LUIS box and all other controlling softwares. For automotive application it works on CAN protocol of J1939 SAE standard. CAN is a two wire twisted pair protocol and it is a standard serial bus, responsible for communication between the ECM and the Doser.

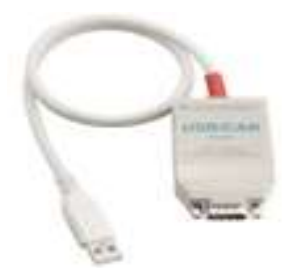

Figure-8 CAN Adapter 


\section{International Journal of Innovative Research in Electrical, Electronics, Instrumentation and Control Engineering}

\section{ISO 3297:2007 Certified}

Vol. 5, Issue 5, May 2017

The electrical continuity fault condition can be simulated with the Digital FMET Breakout box. But the challenges w.r.to Functionality Fault Conditions, which involves the corresponding loads and their connectors to be operated manually. Moreover, there are no programmable output pins available in the LUIS Box. Therefore we have developed new electrical circuits which involves the:

a) Static Relays,

b) Transistor,

c) Electronically Controlled Solenoid Valves,

d) Corresponding mating connectors,

e) Resistor Load Box

Figure-9 shows the In-house developed Integrated Relay board circuit.

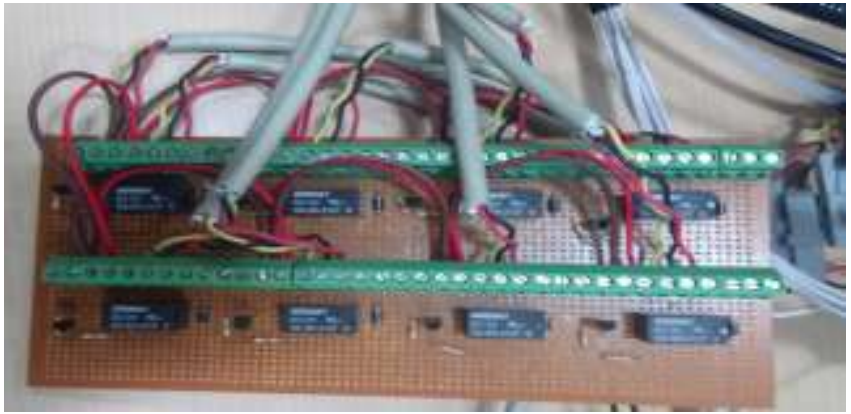

Figure-9 Integrated Relay circuit board

Thus, In-house Relay circuit board has been developed to automatic simulate the functionality fault conditions. The above integrated circuits replaces the required manual actions for creating fault conditions w.r.t. ASOV, BPV, IMV, Nozzle, Air \& DEF lines. Figure-10 shows the automated test bench setup for FMET \& Regression

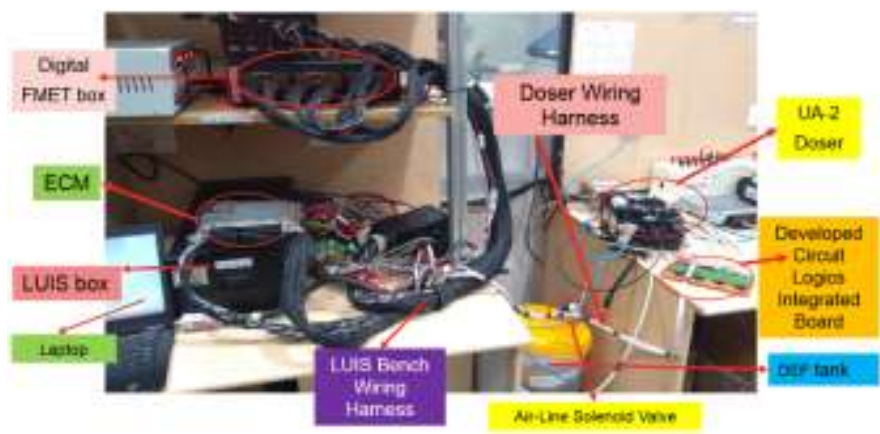

Figure-10 Automated test bench setup using LUIS hardware

\section{IMPLEMENTATION \& RESULTS}

The conventional manual test bench consists of the ECU, Doser, Manual breakout box and the corresponding wiring harness. In fully automated test bench manual break out box is replaced by the Digital FMET box. In addition to these, test sequence scripts are written according to the FMET procedure in the NI- Teststand. Before running the main sequence file of the test script we need to do the Total Bench Configuration (TBC). For doing TBC it is required to prepare certain Tables for the definition of complex wiring harness interconnections relationship among the components viz. ECM, Doser Module, Sensors, Actuators, Digital FMET Box, LUIS Box, Signals and Computer. Thus suitable configuration and calibration files are provided to perform the total bench configuration action. After doing TBC the setup is ready to perform the test. As a case study Pump unit temperature sensor fault code is considered and the code is written in the NI Teststand. The algorithm for simulating the pump unit temperature sensor short to battery positive fault code i.e. creating \& removal of the fault code is shown in the figure- 11 .

The first step is to configure the complete bench setup by doing total bench configuration by using required calibration and the configuration files. The next step is to establish the session by writing scripts in the Teststand. Next step is to open the Feature test report sheet. It is generated automatically showing the behaviour and the status of the fault code. If the fault code appears then it is automatically updated in the test report sheet. If the Actual value is equal to the Target value it shows the PASS status in the feature test report sheet and if the actual value is not equal to the target 
value it will show FAIL status in the feature test report sheet. The next step is to call the sequence file for the Pump unit temperature sensor which contains the step by step procedure to simulate the fault code. The next step is to start the normal dosing operation for priming, purging operation of the Doser to ensure that it is working fine after that it will engage the corresponding relay in the Digital FMET box to simulate the fault code.

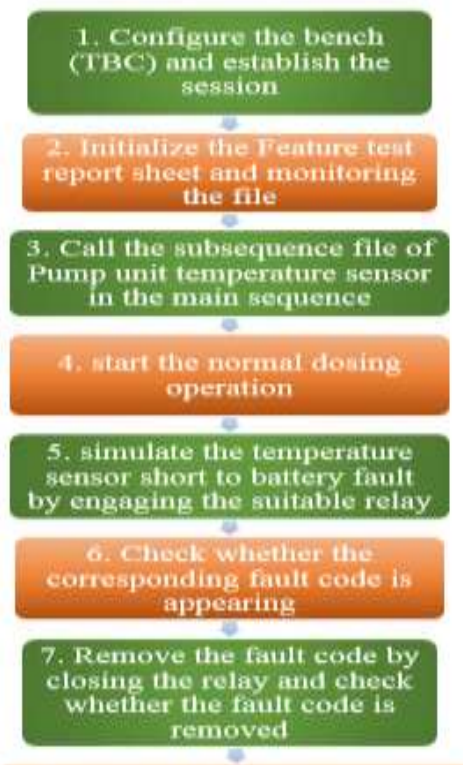

8. Tum Oन the Normal Dosing

Figure-11 Algorithm for simulating temperature sensor short to battery positive.

If the fault code is ACTIVATED, it will return value 1 and the PASS status will get updated in the feature test report sheet. After that the code for removing of the fault is simulated. After this simulation Fault code is removed returning value 2 and again the PASS status gets updated in the feature test report sheet as shown in figure-12

\begin{tabular}{|c|c|c|c|c|c|}
\hline $\begin{array}{l}\text { Checking whether Fault } \\
\text { Code is appearing }\end{array}$ & 1 & -1 & +1.0 & Pass & 0 \\
\hline $\begin{array}{l}\text { CHECKING FAULT CODE } \\
\text { STATUS AFTER } \\
\text { REMOVMA S SHORT }\end{array}$ & & & & & \\
\hline $\begin{array}{l}\text { Checking whether Fault } \\
\text { Code is appearing }\end{array}$ & 2 & $=2$ & +1.0 & Pass & 0 \\
\hline
\end{tabular}

Figure-12: Activation \& Deactivation of the fault code result status

The 'Pass' status shows the Fault Code simulation is achieved successfully. To simulate the functionality fault conditions automatically we have developed the integrated relay circuit board. The block diagram for the connection to simulate functionality fault code is shown in figure-13.

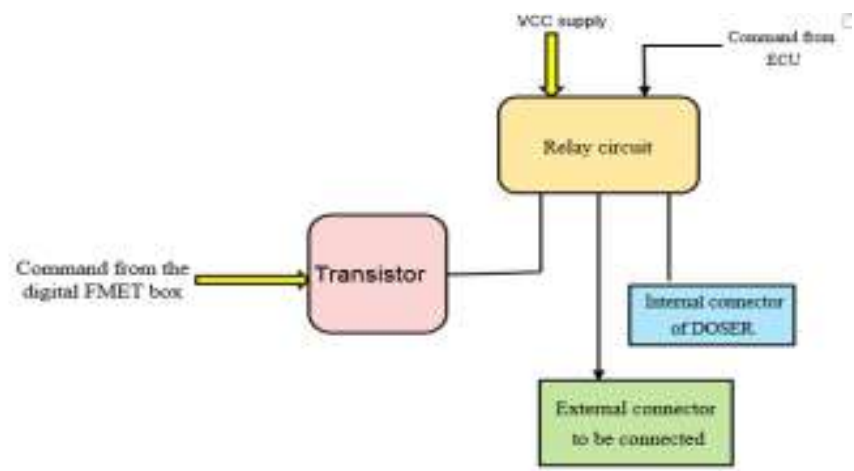

Figure-13 Block diagram to simulate functionality fault conditions 


\section{ISO 3297:2007 Certified}

Vol. 5, Issue 5, May 2017

By using this circuit diagram, we will simulate the functionality fault conditions of manually removal of various actuators. The challenge is that Internal connector/ valve is to be removed and external valve with different voltage required $0 \mathrm{~V}, 6 \mathrm{~V}$ is to be connected which is controlled with the relay circuit. To simulate the fault codes like DEF suction line broken or DEF transfer line block/ broken. Solenoid valves are integrated in the circuit. These valves are controlled through the LUIS box commands and simulate the fault code. The block diagram to simulate Air Line, DEF suction line and DEF transfer line fault code is shown in figure-14.

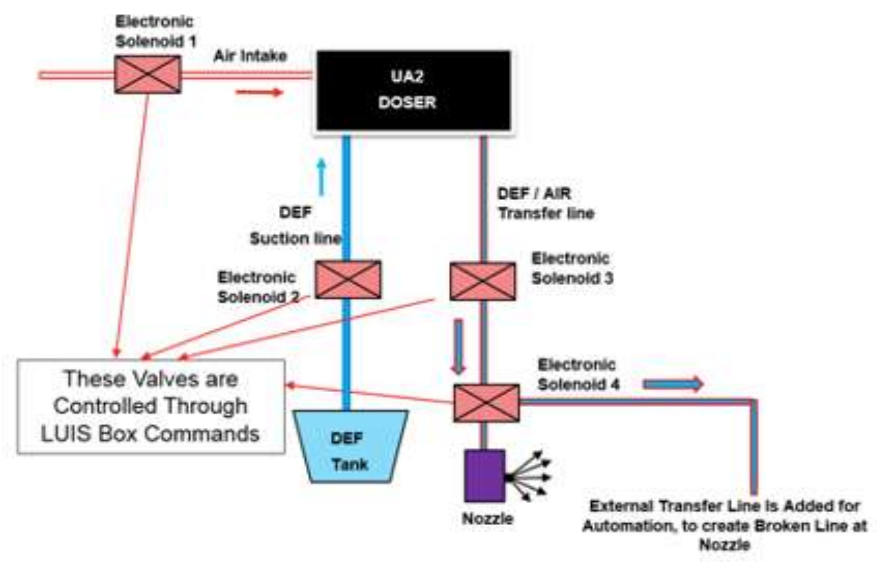

Figure-14 Block diagram to simulate Air, Transfer \& Suction lines functionality fault conditions

\section{CONCLUSION}

The developed HIL system gives Fast Delivery of Test Results, Reduced Manual Errors, Man-Power and Cost Saving, through which On-Time Delivery and Right First Time can be enhanced.Total Time Savings(w.r.to Manual Testing) $\rightarrow 25.94 \%$. Also, it is not required to frequently monitorthe system unless the system is paused due to some errors thus this hardware in loop testing performed in the reproducible, controlled, efficient and effective way creating the real time environment.

\section{ACKNOWLEDGMENT}

The author would like to give her sincere thanks to Head of Department Dr. Daruwala for his valuable references and support throughout the project work. The author would like to thank Prof. Dr. R. N. Awale for his support, cooperation and valuable suggestions.

The author would like to give sincere thanks to her Industrial Guide Chidambar Rao Bhukya and Project sponsor Parag M. Potdar from Cummins Technologies India Pvt. Limited for sharing their valuable knowledge and guiding throughout the project implementation.

\section{REFERENCES}

[1] P. Baracos, G. Murere, C. Rabbath, and W. Jin, "Enabling pc-based HIL simulation for automotive applications," in Proc. IEEE IEMDC, Jun.2001, pp. 721-729.

[2] Mr. Abhijeet Taksale, Vishwas Vaidya, Priti Sahane, Goutham Dronamraju, Vivek Deulkar, "Low cost Hardware-In-Loop for automotive application," IEEE international conference on Industrial instrumentation and control(ICIC), pp, 1109-1114, may2015

[3] Renuka M. Kulkarni, Rohita. P. Patil, Chidambar Rao Bhukya,"Hardware-In-Loop Test Bench Based Failure Mode Effects Test Automation," INTERNATIONAL JOURNAL OF INNOVATIVE RESEARCH IN ELECTRICAL, ELECTRONICS, INSTRUMENTATION AND CONTROL ENGINEERING Vol. 4, Issue 6, June 2016

[4] Sheran Alleset, al., "Real time Hardware in the loop vehicle Simulation," Proceedings of IEEE, 1992.

[5] B. Lu, X. Wu, and A. Monti, "Implementation of a low-cost real- time virtue test bed for hardware-in-the-loop testing," in Proc. IEEE IECON,Nov. 2005, pp. 239-244.

[6] Isermann R, et. al. "Hardware-in-the-loop simulation for the design and testing of engine-control systems,"Control Engineering Practice, 1999.

[7] Liu Y, et.al. “A novel approach to power quality assessment: real time hardware-in-the-loop test bed," IEEE Transaction Power Delivery, 2005.

[8] Jason J. Poon, Michel A. Kinsy, Nathan A. Pallo, Srinivas Devadas, Ivan L. Celanovic "Hardware-in-the-Loop Testing for Electric VehicleDrive Applications," Proceedings of IEEE, 2012.

[9] D. Maclay, "Simulation gets into the loop", IEE Review, vol. 43, no. 3, pp. 109-112, 1997.

[10] Deepa Ramaswamy. et. al., "A case study in hardware-in-the-loop Testing: development of an ECU for a hybrid electric vehicle," proceedings of SAE, 2004-01-0303.

[11] http://www.gartechenterprises.com/luisnextgen_v2.0.pdf

[12] http://www.hil-simulation.com/home/hil-testing.html 